\title{
Predictors of hypertension urgency in primary aldosteronism patients during the first 24 hours after surgery
}

\author{
Juping Zhao ${ }^{1, *}$, Jun Dai, ${ }^{1,}$, Wenlong Zhou ${ }^{1}$, Haofei Wang ${ }^{1}$, Wenbin Rui ${ }^{1}$, Wei He ${ }^{1}$, \\ Zhe Zhu ${ }^{2,3}$, Yu Zhu ${ }^{1}$, Danfeng $X u^{1}$ and Fukang Sun ${ }^{1}$ \\ ${ }^{1}$ Shanghai JiaoTong University School of Medicine, Ruijin Hospital, Department of Urology, Shanghai, 200025 China \\ ${ }^{2}$ Department of Medicine, Division of Regenerative Medicine, University of California, San Diego, School of Medicine, La Jolla, \\ CA, 92037 USA \\ ${ }^{3}$ Department of Stem Cell Biology and Regenerative Medicine, Lerner Research Institute, Cleveland Clinic, Cleveland, OH, \\ 44195 USA \\ "These authors contributed equally to this work
}

Correspondence to: Fukang Sun, email: sfk10570@rjh.com.cn

Keywords: aldosterone-producing adenoma, predictor, hypertension urgency, laparoscopic adrenalectomy

Received: July 04, 2017 Accepted: September 21, 2017 Published: October 07, 2017

Copyright: Zhao et al. This is an open-access article distributed under the terms of the Creative Commons Attribution License 3.0 (CC BY

3.0), which permits unrestricted use, distribution, and reproduction in any medium, provided the original author and source are credited.

\section{ABSTRACT}

Study about blood pressure variation in the first 24 hours post-operation is limited in patients with adrenal aldosterone-producing adenoma. We aim to evaluate the potential predictors for postoperative hypertension urgency during the first 24 hours after laparoscopic adrenalectomy in patients with aldosterone-producing adenoma. Clinical data of 177 patients with aldosterone-producing adenoma were retrospectively collected from January 2009 to December 2015 and the potential factors that may influence postoperative blood pressure during the first $\mathbf{2 4}$ hours after surgery were analyzed. The factors included gender, age, body mass index, preoperative maximum systolic blood pressure, number of antihypertensive medicines, preoperative spironolactone treatment, duration of hypertension, surgical method and approach, adenoma diameter, preoperative proteinuria, estimated glomerular filtration rate, serum potassium and serum aldosterone. Univariate and multivariate regression analyses were used to evaluate the relationship between the above variables and postoperative hypertension urgency. We found that the proportion of patients with a higher systolic blood pressure $\geq 160 \mathrm{mmHg}$ and $\geq 180 \mathrm{mmHg}$ were significantly increased post-operation (both $p<0.001$ ). In multivariate analysis, the maximum systolic blood pressure was an independent predictor of postoperative hypertension urgency, and the cut-off point was $157 \mathrm{mmHg}$ with the sensitivity of $66 \%$ and specificity of $82 \%$. Multivariable analysis also showed that preoperative maximum systolic blood pressure and number of antihypertensive medicines were independent risk factors for higher postoperative systolic blood pressure. This study was derived from a high volume adrenal tumor center, and these data may provide a potential tool to guide preoperative counseling.

\section{INTRODUCTION}

Among the general hypertensive population, primary hyperaldosteronism (PHA) is widely recognized as the most common cause of secondary hypertension [1]. About $3-10 \%$ of hypertension patients are diagnosed with PHA $[2,3]$, with a higher rate of cerebrovascular complications
[4]. Therefore, PHA is a recent research hotspot. PHA is caused by the hypersecretion of aldosterone hormone due to adrenocortical lesions, and is associated with clinical manifestations of hypertension and hypokalemia. There are two subtypes of PHA, aldosterone-producing adenoma (APA) and idiopathic hyperaldosteronism (or bilateral adrenal hyperplasia). APA accounted for about $60 \%$ of PHA 
[5]. Hypokalemia can be successfully cured in most APA patients by surgery, which includes partial adrenalectomy and unilateral total adrenalectomy [6, 7]. However, the recovery of blood pressure(BP) is still facing challenge in clinical setting. There are significant variations in BP during the first 24 hours after surgery, which can cause acute severe cerebrovascular complications, such as stroke, cardiac infarction, etc.

Numerous studies have identified factors associated with long-term outcomes after adrenalectomy for PHA [8-10]. However, knowledge of BP variations in the first 24 hours post-operation is limited. In this study, we evaluated the potential predictors for postoperative hypertension urgency during the first 24 hours after laparoscopic adrenalectomy (LA) in patients with APA.

\section{RESULTS}

\section{Clinical demographics}

A total of 177 patients with APA were enrolled in this study. The demographic characteristics of the patients are shown in Table 1 . The median age was 46 (IQR = $38-53$ ) years, and $44.6 \%$ of the patients were male. The median duration of preoperative hypertension was 5.5 years $(\mathrm{IQR}=2-10$ years $)$. Preoperative evaluation indicated that the patients were on a median of two antihypertensive medicines, with a median maximum systolic blood pressure(SBPmax) of $140 \mathrm{mmHg}(\mathrm{IQR}=130-158 \mathrm{mmHg})$.

Laparoscopic partial or total adrenalectomy was successfully performed in all patients, $61.6 \%$ and $38.4 \%$, respectively, without conversions to open surgery. Surgery was conducted by the transperitoneal approach in 39.5\% of cases and retroperitoneal approach in $60.5 \%$ of cases. All 177 cases were pathologically confirmed to be benign adrenal cortical adenoma. There were no deaths or severe complications during hospitalization. The $\mathrm{CT}$, specimen and pathology images of a typical APA case are shown in Figure 1.

The median concentration of serum aldosterone at diagnosis was $466 \mathrm{pg} / \mathrm{mL}(\mathrm{IQR}=306-700)$. The normal value of serum aldosterone at our center was 38-313 $\mathrm{pg} / \mathrm{ml}$ in standing position. Thirty-one patients $(17.5 \%)$ received intravenous antihypertensive medication in the immediate postoperative period because of persistent excessively high BP.

\section{Trend of blood pressure variations}

The systolic blood pressure(SBP) increased by a median of $20 \mathrm{mmHg}$ (IQR $=5-28 \mathrm{mmHg}$ ) post-operation, and the median percentage was $13.4 \%(\mathrm{IQR}=3.3-20.4 \%)$. There were 91 patients (51.4\%) with $\mathrm{BP} \geq 160 \mathrm{mmHg}$ postoperation as compared to 40 patients (22.6\%) pre-operation, $p<0.01$. Hypertension urgency was seen in $29(16.4 \%)$ patients after surgery, with a significant increase from 11 cases $(6.2 \%)$ pre-operation.

\section{Factors associated with hypertension urgency in the first 24 hours post-operation}

When postoperative hypertension urgency was considered as a categorical variable for univariate analysis, preoperative SBPmax ( $p=0.001$ ), duration of preoperative hypertension $(<5$ years $)(p=0.001)$, surgical method $(p=0.043)$, and preoperative serum potassium $(<3.5$ $\mathrm{mmol} / \mathrm{L})(p=0.010)$ were associated with postoperative hypertensive urgency (Table 2).

In multivariate analysis, preoperative SBPmax ( $p$ $=0.001)$ was the only independent prognosticator (HR: $0.180,95 \%$ confidence interval $0.079-0.281)$. When the ROC curve of preoperative SBPmax was generated, the area under the curve was 0.771 , consistent with good fit to the postoperative hypertension urgency data (Figure 2). The preoperative SBPmax of $157 \mathrm{mmHg}$ was the cut-off point, with $65.5 \%$ sensitivity and $82.4 \%$ specificity for postoperative hypertension urgency.

On univariate analysis, preoperative $\operatorname{SBPmax}(p$ $=0.000)$, number of antihypertensive medications $(p=$ $0.032)$, duration of preoperative hypertension $(<5$ years $)$ $(p=0.007)$, and serum potassium $(<3.5 \mathrm{mmol} / \mathrm{L})(p=$ $0.015)$ were associated with postoperative higher BP as a continuous variable (Table 3). Multivariate logistic regression analysis was performed on factors identified in univariate analysis, and showed that preoperative SBPmax $(p=0.045)$ and number of antihypertensive medications $(p=0.023)$ were independent prognosticators.

\section{DISCUSSION}

Hypertension can cause numerous comorbidities, and is the leading preventable risk factor for death in recent decades $[11,12]$. PHA is widely recognized as one of the most common causes of secondary hypertension, and persistent hypertension causes targeted organ dysfunction [4] . Monticone and colleagues reported that 5.9\% patients were diagnosed with PHA among 1,672 primary hypertension patients [1]. APA is a subtype of PHA that can be cured by adrenalectomy [6,7]. Most studies focus on the predictive factors of long-term outcomes after adrenalectomy [13, 14]. Older age, longer duration of hypertension, increased serum creatinine, preoperative use of $>2$ antihypertensive medicines, and positive family history of hypertension were reported as predictors of persistent hypertension after adrenalectomy in PHA population [13, 14]. Clinically, BP varies significantly during the first 24 hours post-operation. To our knowledge, in the setting of APA, BP variations during the first 24 hours post-operation has not been well studied. Therefore, we conducted this study to evaluate the potential predictors for postoperative hypertension urgency within the first 24 hours after LA in patients with APA.

High BP induced by hyperaldosteronism can be exacerbated in the immediate postoperative period by 
Table 1: Characteristics of 177 patients with adrenal aldosterone-producing adenoma

\begin{tabular}{ll}
\hline \multicolumn{1}{c}{ Variable } & \multicolumn{1}{c}{ Value } \\
\hline No. of males (\%) & $79(44.6 \%)$ \\
Age of operation (years) (Median, IQR) & $46(38-53)$ \\
BMI $\left(\mathrm{kg} / \mathrm{m}^{2}\right)$ (Median, IQR) & $23.4(21.5-25.5)$ \\
Duration of hypertension (years) (Median, IQR) & $5.5(2.0-10.0)$ \\
Preoperative SBPmax (mmHg) (Median, IQR) & $140(130-158)$ \\
Number of preoperative antihypertensive medicine(Median, IQR) & $2(2-3)$ \\
Surgery type & \\
$\quad$ Partial adrenalectomy & $109(61.6 \%)$ \\
$\quad$ Total unilateral adrenalectomy & $68(38.4 \%)$ \\
Surgery approach & \\
$\quad$ Transperitoneal & $70(39.5 \%)$ \\
$\quad$ Retroperitoneal & $107(60.5 \%)$ \\
Operating time (min) (Median, IQR) & $105(85-130)$ \\
Adenoma diameter (cm) (Median, IQR) & $1.5(1.2-2.0)$ \\
Preoperative eGFR (mL/min/1.73m²) (Median, IQR) & $102(84-118)$ \\
Preoperative serum aldosterone (ng/dL) (Median, IQR) & $466(306-700)$ \\
Preoperative serum potassium (mmol/L) (Median, IQR) & $3.9(3.6-4.1)$ \\
Postoperative blood pressure (mmHg) (Median, IQR) & $160(150-170)$ \\
\hline
\end{tabular}

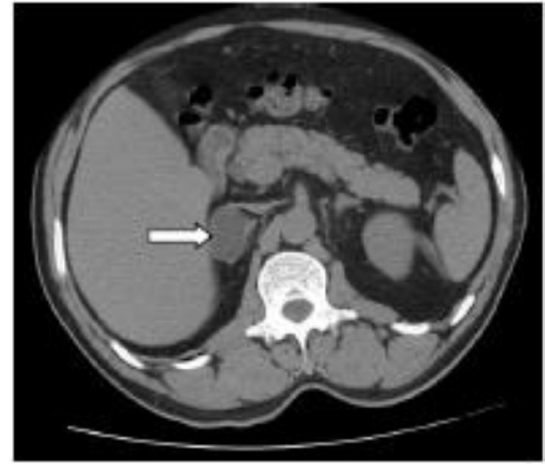

A

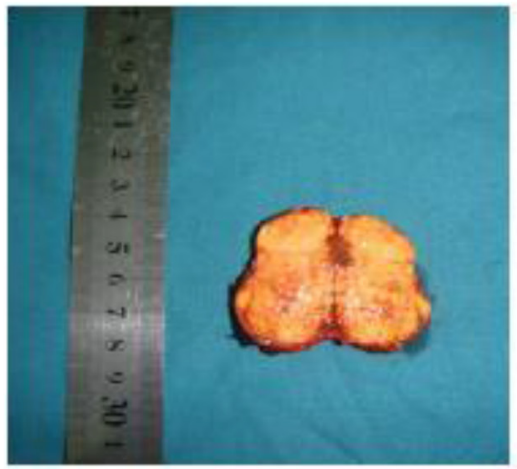

D

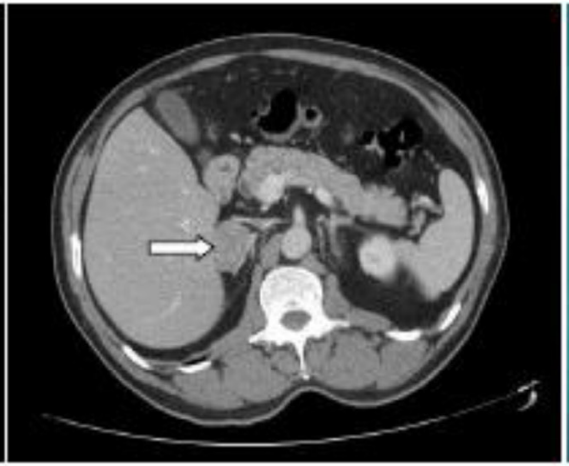

B

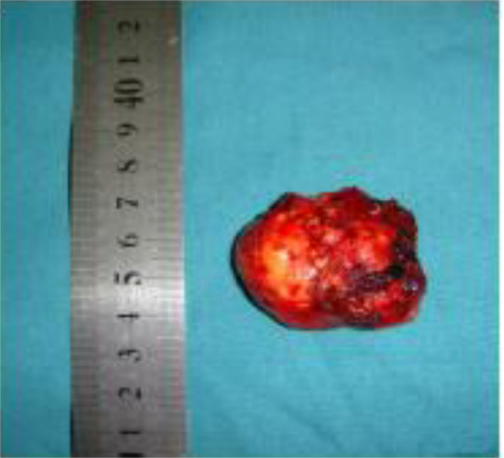

C

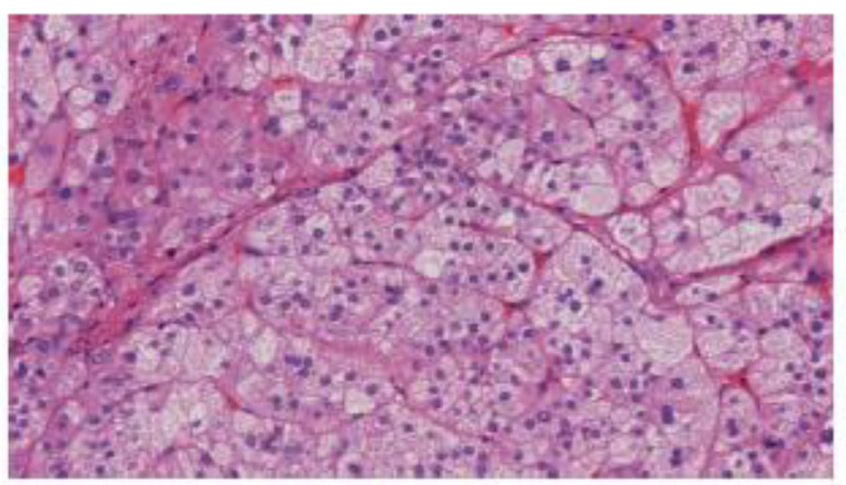

E

Figure 1: Primary hyperaldosteronism in a 59-year-old male patient. (A and B) CT image (plain and contrast) of primary adrenal aldosterone-producing adenoma. (C and D) Specimen picture of aldosterone-producing adenoma. (E) H \& E staining of the aldosterone-producing adenoma $(10 \times 10)$. 
Table 2: Univariate analysis of risk factors for postoperative hypertension urgency

\begin{tabular}{lccc}
\hline \multicolumn{1}{c}{ Variable } & NPHU group & PHU group & $\boldsymbol{P}$ \\
\hline Male & $67 / 148(45.3 \%)$ & $12 / 29(41.4 \%)$ & 0.702 \\
Age of operation (years) (Median, IQR) & $46(38-52)$ & $50(39-56)$ & 0.388 \\
BMI $\left(\mathrm{kg} / \mathrm{m}^{2}\right)$ (Median, IQR) & $23.5(21.6-25.4)$ & $22.7(21.5-26.5)$ & 0.625 \\
Preoperative SBPmax (mmHg) (Median, IQR) & $140(130-150)$ & $160(150-173)$ & $\mathbf{0 . 0 0 1}^{* *}$ \\
Number of preoperative antihypertensive medicine & $2(2-3)$ & $2(2-3)$ & 0.393 \\
(Median, IQR) & $126 / 148(85.1 \%)$ & $23 / 29(79.3 \%)$ & 0.435 \\
Preoperative spirolactone treatment & $72 / 148(48.6 \%)$ & $4 / 29(13.8 \%)$ & $\mathbf{0 . 0 0 1}^{* *}$ \\
Duration of hypertension $<5$ years & $96 / 148(64.9 \%)$ & $13 / 29(44.8 \%)$ & $\mathbf{0 . 0 4 3}^{*}$ \\
Surgical method, partial & $59 / 148(39.9 \%)$ & $11 / 29(37.9 \%)$ & 0.847 \\
Surgical approach, transperitoneal & $1.5(1.2-2.0)$ & $1.5(1.2-2.0)$ & 0.856 \\
Adenoma diameter (cm) (Median, IQR) & $5 / 148(3.4 \%)$ & $2 / 29(6.9 \%)$ & 0.377 \\
Preoperative proteinuria & $101(83-115)$ & $111(88-124)$ & 0.251 \\
Preoperative eGFR (ml/min/1.73 m²) (Median, & & & \\
IQR) & $17 / 148(11.5 \%)$ & $9 / 29(31.0 \%)$ & $\mathbf{0 . 0 1 0}^{*}$ \\
Preoperative serum potassium $(\mathrm{mmol} / \mathrm{L})$ & $467(294-686)$ & $451(342-766)$ & 0.215 \\
(<3.5 mmol/L) & & \\
Preoperative serum aldosterone $(\mathrm{pg} / \mathrm{ml})$ & &
\end{tabular}

NPHU: non-postoperative hypertensive urgency; PHU: postoperative hypertensive urgency. ${ }^{*} p<0.05,{ }^{* *} p<0.01$

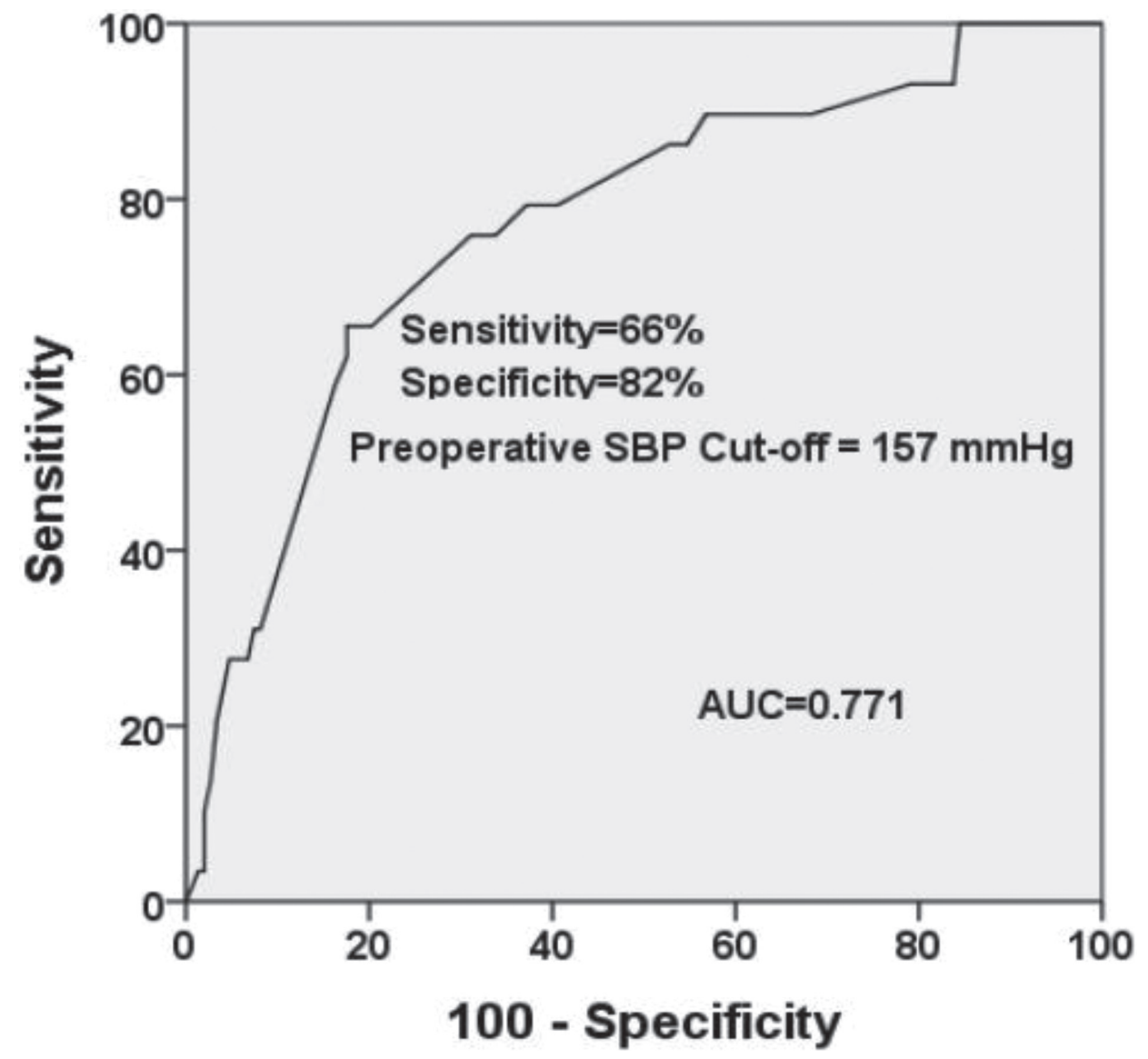

Figure 2: ROC curve indicated that preoperative SBPmax can accurately predict the occurrence of postoperative hypertensive urgency. The cut-off point was $157 \mathrm{mmHg}$, with the sensitivity of $66 \%$ and specificity of $82 \%$. 
Table 3: Univariate analysis of risk factors for higher postoperative SBP

\begin{tabular}{lcc}
\hline \multicolumn{1}{c}{ Variable } & Value & $\boldsymbol{P}$ \\
\hline Male & $44.6 \%$ & 0.161 \\
Age of operation (years) (Median, IQR) & $46(38-53)$ & 0.081 \\
BMI $\left(\mathrm{kg} / \mathrm{m}^{2}\right.$ ) (Median, IQR) & $23.4(21.5-25.5)$ & 0.351 \\
Preoperative SBPmax (mmHg) (Median, IQR) & $140(130-158)$ & $\mathbf{0 . 0 0 0}^{* *}$ \\
Number of preoperative antihypertensive medicine (Median, IQR) & $2(2-3)$ & $\mathbf{0 . 0 3 2}^{*}$ \\
Preoperative spirolactone treatment & $84.2 \%$ & 0.224 \\
Duration of hypertension (<5 years) & $42.9 \%$ & $\mathbf{0 . 0 0 7}^{* *}$ \\
Surgical method (partial) & $61.6 \%$ & 0.140 \\
Surgical approach (transperitoneal) & $39.5 \%$ & 0.445 \\
Adenoma diameter (cm) (Median, IQR) & $1.5(1.2-2.0)$ & 0.421 \\
Preoperative proteinuria & $4 \%$ & 0.294 \\
eGFR (ml/min/1.73 m ${ }^{2}$ ) (Median, IQR) & $102(84-118)$ & 0.883 \\
Preoperative serum potassium $(<3.5 \mathrm{mmol} / \mathrm{L})$ & $14.7 \%$ & $\mathbf{0 . 0 1 5 ^ { * }}$ \\
Preoperavite serum aldosterone $(\mathrm{pg} / \mathrm{ml})$ & $466(306-700)$ & 0.275 \\
\hline
\end{tabular}

${ }^{*} p<0.05,{ }^{* *} p<0.01$.

factors such as pain, hypercapnia, hypoxia, intravascular volume overload and hyperthermia $[15,16]$. In addition, oral antihypertensives are contraindicated during the first 24 hours post-operation due to temporary impairment of digestive function. Therefore, postoperative hypertension urgency was frequently observed after laparoscopic adrenalectomy in patients with APA. Though the progress of anesthesia was fixed and similar at our center and adrenalectomy was performed by experienced surgeons, the median percentage of $\mathrm{SBP} \geq 160 \mathrm{mmHg}$ increased significantly from $22.6 \%$ pre-operation to $51.4 \%$ post-operation. The median percentage of $\mathrm{SBP} \geq 180 \mathrm{mmHg}$ increased from $6.2 \%$ to $16.4 \%, p<$ 0.01 . In the 29 patients with $\mathrm{SBP} \geq 180 \mathrm{mmHg}$ postoperation, intravenous antihypertensive drugs were immediately administered when oral therapy could not be administered. Nicardipine Hydrochloride injection (0.5-6 ug/ $\mathrm{kg} / \mathrm{min})$ or Urapidil Hydrochloride injection (2 $\mathrm{mg} / \mathrm{min}$ ) were the primary therapeutic options at our institution. Drip speed adjustment was made according to the real-time SBP. Timely intervention led to safe and efficient outcome, without cerebrovascular complications.

There was a trend of increase in BP after surgery due to several factors. Wachtel and colleagues evaluated several protective factors of persistent postoperative hypertension, including female gender, BMI $(<25 \mathrm{~kg} /$ $\left.\mathrm{m}^{2}\right)$, hypertension $(<5$ years duration), serum creatinine $(<0.8 \mathrm{mg} / \mathrm{dL})$, and $<2$ preoperative antihypertensive medicine [8]. In this study, we evaluated the potential factors that could increase the postoperative SBP within 24 hours in patients with APA. In the multivariable analysis, using postoperative SBPmax as a continuous variable, preoperative SBPmax and number of antihypertensive medicines were the independent prognosticators, HR 6.295 and 3.387, respectively (both $p<0.05$ ). The number of antihypertensive medicines was a meaningful parameter to predict the immediate and longterm BP variations. Considering SBPmax $\geq 180 \mathrm{mmHg}$ as a categorical variable (whether hypertension urgency or not), only preoperative SBPmax was an independent prognosticator, $p=0.001$.

Actually, there are many additional factors which could affect BP variation after surgery, such as anesthesia. In our study, the BP influence of anesthesia exists objectively, though the progress of anesthesia was fixed and similar. This is one of the limitation

SBP is considered as an important parameter in the hypertensive population with prevalent cerebrovascular diseases [17]. Recent studies provided clinical evidence for a target SBP in elderly patients that was significantly associated with high risk of cardiovascular events [18]. So we propose preoperative SBPmax as a potential predictor of hypertension urgency in patients with APA within the first 24 hours post-operation. Additionally, we generated the ROC curve for further analysis and found that preoperative SBPmax of $157 \mathrm{mmHg}$ was the cut-off point, whose sensitivity was $66 \%$ and specificity was $82 \%$.

In our study, gender, BMI, duration of hypertension and preoperative serum creatinine were not significantly associated with postoperative hypertension urgency in multivariate analysis. The morbidity of APA was similar between men and women in our cohort, and there was no significant variation in BMI (median 23.4, IQR:21.5-25.5) due to Asian ethnicity. Interestingly, we found that duration of hypertension was not a predictor for postoperative 
immediate SBP, which was inconsistent with previous reports on long-term hypertension [13, 14]. Due to limited sample size in our study, further research is needed in this field, and post-operative BP should be followed in the longer term in these patients.

In conclusion, our data derived from the high volume adrenal tumor center and the results are relatively representative in population. The preoperative SBPmax was independently associated with hypertension urgency within the first 24 hours after laparoscopic adrenalectomy. These data may provide a potential tool to guide preoperative counseling. Control of preoperative SBP could decrease the occurrence of hypertension urgency within the first 24 hours post-operation.

\section{MATERIALS AND METHODS}

\section{Study population}

After approval by the Ethics Committee of Ruijin Hospital, the adrenal surgery registry database was used to identify patients who were managed with LA from January 2009 to December 2015. During this time, clinical data of 177 patients with APA were retrospectively collected at our adrenal disease center. Written informed consent was obtained from all enrolled patients. Before surgery, the patients underwent an examination in the department of Hypertension or Endocrinology. The diagnosis of PHA was based on endocrinological evaluation, preoperative imaging studies (CT or MRI) and postoperative pathology. Before endocrinological evaluation, mineralocorticoid receptor antagonists were washed-out for at least six weeks. Other antihypertensive drugs were replaced with verapamil (Isoptin) sustained-release tablets for at least two weeks. A positive diagnosis for PHA was based on elevated serum aldosterone level with increased aldosterone-to-renin ratio, and was confirmed by the absence of aldosterone suppression after a saline-loading test. All serum aldosterone concentrations were measured in a standing position, with normal value of $38-313 \mathrm{pg} / \mathrm{mL}$ at our center. Adrenal venous sampling (AVS) was conducted to distinguish between unilateral and bilateral adrenal lesions. Patients who refused to undergo AVS were excluded from this study. Each paraffin section and hematoxylin-eosin (HE) stained section was independently evaluated by two pathologists using standard criteria based on the WHO classification. The third independent pathologist adjudicated the section in case of different opinions.

\section{Surgery}

Laparoscopic total or partial adrenalectomy was performed via a standard transperitoneal approach in 70 patients and a retroperitoneal approach in 107 patients. The decision to perform a partial or total unilateral adrenalectomy was intraoperatively made based on the position of the adenoma in the adrenal gland and whether concomitant multiple microadenomas and nodules were presented $[19,20]$. Laparoscopic partial adrenalectomy was preferred when the adenoma was eccentric, without multiple microadenomas and nodules. In laparoscopic partial adrenalectomy, the adrenal central vein was preserved if possible to ensure sufficient blood drainage to the remnant gland. Laparoscopic total unilateral adrenalectomy was imperative when the adenoma was near the core of the gland or when multiple microadenomas and nodules were detected. In this study, the operation time covered the period from anesthesia start to anesthesia recovery.

\section{Potential risk factors}

The potential risk factors included gender, age, body mass index (BMI), preoperative maximum systolic blood pressure (SBPmax), number of antihypertensive medicines, preoperative spironolactone treatment, duration of hypertension, surgical method and approach, adenoma diameter, preoperative proteinuria, estimated glomerular filtration rate (eGFR), serum potassium and serum aldosterone. BP was measured by the adjusted electronic sphygmomanometer routinely in our ward at least three times a day before surgery and then noted in the medical records. The preoperative highest SBP value was considered as a potential risk factor. Postoperative SBP was obtained via electrocardiogram monitors (Boeblingen $\mathrm{GmbH}$, Philips Inc., Germany) as soon as the patients were transferred back to the wards during the first 24 hours post-operation. Semiautomatic noninvasive measurements of BP were performed every half an hour in the supine position. In this study, hypertension urgency was defined as $\mathrm{SBP} \geq 180 \mathrm{mmHg}$ [21], which typically results in headache, dizziness, vomiting and convulsions. Postoperative hypertension was considered if SBP $\geq$ $160 \mathrm{mmHg}$. We checked the patient's medical history to determine the number of antihypertensive medicines, whether or not preoperative spironolactone treatment was administered, and duration of hypertension. Proteinuria was defined as a urine protein concentration $>30 \mathrm{mg} / \mathrm{dL}$, and eGFR was calculated using the following equation developed and validated based on Chinese population: eGFR $\left(\mathrm{mL} / \mathrm{min} / 1.73 \mathrm{~m}^{2}\right)=175 \times($ serum creatinine $)-1.234$ $\times$ (age) $-0.197(\times 0.75$ in case of women) [22]. Preoperative serum potassium and aldosterone levels were detected by routine lab tests at our institute.

\section{Statistical analysis}

Group comparisons were made using the Students' $t$-test, Chi-square test, or Wilcoxon rank-sum test, as appropriate. Univariate analysis of risk factors for postoperative hypertension urgency and higher SBP was performed to detect the significant prognosticator. Then, multivariate analysis was used to evaluate the independent prognosticator. To dichotomize continuous 
variables, receiver operating characteristic (ROC) curves of independent prognosticators were generated, and values with maximal sensitivity were identified. The differences were considered significant if $p<0.05$. Data were analyzed by using SPSS version 20.0 (SPSS Inc., Chicago, IL, USA).

\section{CONFLICTS OF INTEREST}

None.

\section{FUNDING}

This research was funded by National Natural Science Foundation of China (grant number 81572621).

\section{REFERENCES}

1. Monticone S, Burrello J, Tizzani D, Bertello C, Viola A, Buffolo F, Gabetti L, Mengozzi G, Williams TA, Rabbia F, Veglio F, Mulatero P. Prevalence and Clinical Manifestations of Primary Aldosteronism Encountered in Primary Care Practice. J Am Coll Cardiol. 2017; 69:1811-1820.

2. Mulatero P, Stowasser M, Loh KC, Fardella CE, Gordon RD, Mosso L, Gomez-Sanchez CE, Veglio F, Young WF Jr. Increased diagnosis of primary aldosteronism, including surgically correctable forms, in centers from five continents. J Clin Endocrinol Metab. 2004; 89:1045-1050.

3. Williams JS, Williams GH, Raji A, Jeunemaitre X, Brown NJ, Hopkins PN, Conlin PR. Prevalence of primary hyperaldosteronism in mild to moderate hypertension without hypokalaemia. J Hum Hypertens. 2006; 20:129-136.

4. Milliez P, Girerd X, Plouin PF, Blacher J, Safar ME, Mourad JJ. Evidence for an increased rate of cardiovascular events in patients with primary aldosteronism. J Am Coll Cardiol. 2005; 45:1243-1248.

5. Sawka AM, Young WF, Thompson GB, Grant CS, Farley DR, Leibson C, van Heerden JA. Primary aldosteronism: factors associated with normalization of blood pressure after surgery. Ann Intern Med. 2001; 135:258-261.

6. Mattsson C, Young WF Jr. Primary aldosteronism: diagnostic and treatment strategies. Nat Clin Pract Nephrol. 2006; 2:198-208; quiz, 191 p following 230.

7. Funder JW, Carey RM, Fardella C, Gomez-Sanchez CE, Mantero F, Stowasser M, Young WF Jr, Montori VM. Case detection, diagnosis, and treatment of patients with primary aldosteronism: an endocrine society clinical practice guideline. J Clin Endocrinol Metab. 2008; 93:3266-3281.

8. Wachtel H, Cerullo I, Bartlett EK, Kelz RR, Cohen DL, Karakousis GC, Roses RE, Fraker DL. Long-term blood pressure control in patients undergoing adrenalectomy for primary hyperaldosteronism. Surgery. 2014; 156:13941402; discussion 1402-1393.

9. Mulatero P, Monticone S, Bertello C, Viola A, Tizzani D, Iannaccone A, Crudo V, Burrello J, Milan A, Rabbia F,
Veglio F. Long-term cardio- and cerebrovascular events in patients with primary aldosteronism. J Clin Endocrinol Metab. 2013; 98:4826-4833.

10. Catena C, Colussi G, Lapenna R, Nadalini E, Chiuch A, Gianfagna P, Sechi LA. Long-term cardiac effects of adrenalectomy or mineralocorticoid antagonists in patients with primary aldosteronism. Hypertension. 2007; 50:911-918.

11. Sheng CS, Liu M, Kang YY, Wei FF, Zhang L, Li GL, Dong Q, Huang QF, Li Y, Wang JG. Prevalence, awareness, treatment and control of hypertension in elderly Chinese. Hypertens Res. 2013; 36:824-828.

12. He J, Gu D, Wu X, Reynolds K, Duan X, Yao C, Wang J, Chen CS, Chen J, Wildman RP, Klag MJ, Whelton PK. Major causes of death among men and women in China. N Engl J Med. 2005; 353:1124-1134.

13. Young WF. Primary aldosteronism: renaissance of a syndrome. Clin Endocrinol (Oxf). 2007; 66:607-618.

14. Meyer A, Brabant G, Behrend M. Long-term follow-up after adrenalectomy for primary aldosteronism. World J Surg. 2005; 29:155-159.

15. Nishigaki R, Ito A, Kamei J, Takahashi T, Fujii E. Risk factors for development of postoperative hypertension. Methods Find Exp Clin Pharmacol. 2001; 23:203-207.

16. Lien SF, Bisognano JD. Perioperative hypertension: defining at-risk patients and their management. Curr Hypertens Rep. 2012; 14:432-441.

17. Mant J, McManus RJ, Roalfe A, Fletcher K, Taylor CJ, Martin U, Virdee S, Greenfield S, Hobbs FD. Different systolic blood pressure targets for people with history of stroke or transient ischaemic attack: PAST-BP (Prevention After Stroke-Blood Pressure) randomised controlled trial. BMJ. 2016; 352:i708.

18. Franco Palacios CR, Haugen EN, Thompson AM, Rasmussen RW, Goracke N, Goyal P. Clinical outcomes with a systolic blood pressure lower than $120 \mathrm{mmHg}$ in older patients with high disease burden. Ren Fail. 2016; 38:1364-1369.

19. Cavallaro G, Letizia C, Polistena A, De Toma G. Laparoscopic adrenal-sparing surgery: personal experience, review on technical aspects. Updates Surg. 2011; 63:35-38.

20. Roukounakis N, Dimas S, Kafetzis I, Bethanis S, Gatsulis N, Kostas H, Kyriakou V, Michas S. Is preservation of the adrenal vein mandatory in laparoscopic adrenal-sparing surgery? JSLS. 2007; 11:215-218.

21. Patel KK, Young L, Howell EH, Hu B, Rutecki G, Thomas G, Rothberg MB. Characteristics and Outcomes of Patients Presenting With Hypertensive Urgency in the Office Setting. JAMA Intern Med. 2016; 176:981-988.

22. Kong X, Ma Y, Chen J, Luo Q, Yu X, Li Y, Xu J, Huang S, Wang L, Huang W, Wang M, Xu G, Zhang L, et al. Evaluation of the Chronic Kidney Disease Epidemiology Collaboration equation for estimating glomerular filtration rate in the Chinese population. Nephrol Dial Transplant. 2013 ; 28:641-651. 